\title{
Article introductif
}

\section{Les transformations du conseil face à une nouvelle agriculture}

\author{
Guy Faure ${ }^{1}$ \\ Claude Compagnone ${ }^{2}$ \\ ${ }^{1}$ Cirad \\ UMR Innovation \\ 79, rue Jean-François Breton \\ TA $60 / 15$ \\ 34398 Montpellier cedex 5 \\ France \\ <guy.faure@cirad.fr> \\ 2 Inra Listo \\ AgroSup Dijon \\ 26, boulevard du Docteur Petitjean \\ BP 87999 \\ 21079 Dijon cedex \\ France \\ $<$ c.compagnone@agrosupdijon.fr>
}

\begin{abstract}
Résumé
La question agricole revient à l'ordre du jour des débats internationaux afin de répondre aux défis alimentaires en inventant de nouvelles formes de production plus durables. Après des années de désengagement des États et de mise en œuvre de politiques de privatisation, le conseil en agriculture connaît un regain d'intérêt. L'objectif de cet article est de positionner les débats auxquels il donne actuellement lieu. Son organisation à l'échelle d'un territoire est le fruit de choix politiques et de rapports sociaux entre acteurs qui ont évolué au cours du temps. Les formes qu'il peut prendre oscillent en permanence entre une logique d'encadrement des agriculteurs et une logique d'accompagnement, souvent portées par des acteurs différents et parfois avec des orientations contradictoires. L'accroissement du nombre des acteurs qui interagissent dans la sphère agricole et rurale, de même que la forte augmentation des risques et incertitudes qui pèsent sur la production agricole, conduisent à s'interroger sur la place du conseil et sur la manière dont il peut s'insérer au sein d'un système d'innovation.
\end{abstract}

Mots clés : conseil agricole ; innovation ; politique agricole.

Thèmes : économie et développement rural ; méthodes et outils.

\section{Abstract \\ Transforming advisory services to meet new farming needs}

Agriculture has once again become a major issue of international debate to both meet food challenges and invent new, more sustainable, forms of production. After years of governmental disinterest and the implementation of policies of privatization, agricultural advisory services is regaining interest. The goal of this article is to position the debates taking place on the issue. The organization of advisory services at the territorial scale is the fruit of policy choices and social relationships between actors which have evolved through time. The forms such intervention can take in connection with farmers, constantly oscillating between logics of managing them and logics of accompanying them, are often born by different and sometimes contradictory actors. The increasing numbers of actors interacting within the farming and rural sphere along with the rapidly increasing risks and uncertainties weighing on agricultural production lead to questions on the role advisory services should play and how it should position itself within an innovation system.

Key words: advisory services; agricultural policies; innovation.

Subjects: economy and rural development; tools and methods.

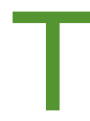
oute une série de transformations conduit aujourd'hui à s'interroger sur la forme que peut prendre le conseil en agriculture. Ces transformations tiennent bien évi- demment aux changements que vivent nos sociétés mais aussi à l'émergence de nouvelles préoccupations et à la place qu'y occupe l'agriculture. Un couplage s'opère entre ces transformations et la 
manière dont le conseil en agriculture peut être pensé et mis en œuvre. Comprendre la nature de ce couplage en se penchant sur la façon dont s'est structuré le conseil, sur la forme qu'il prend actuellement ainsi que sur la manière dont il est pris en charge par différentes institutions, est donc essentiel. Les articles réunis dans ce numéro contribuent, à partir d'angles différents, à apporter un éclairage spécifique sur ces questions. Nous nous proposons dans cet article introductif de faire ressortir les principaux enjeux auxquels le conseil est confronté afin de pouvoir situer la façon dont les contributions qui suivent se positionnent par rapport aux débats actuels sur le conseil.

Nous commencerons par préciser en quoi l'agriculture est redevenue aujourd'hui un enjeu mondial pour voir ensuite comment des formes d'organisation du conseil différentes se sont succédé selon les périodes. Nous poursuivrons en faisant apparaître la dimension politique de l'organisation du conseil et en précisant la manière dont il peut contribuer au développement. Ce cheminement nous conduira à définir les logiques parfois contradictoires qui animent l'activité de conseil. Nous terminerons notre propos en nous intéressant à la manière dont les pouvoirs publics et la recherche se saisissent actuellement des questions de conseil en agriculture. Au regard de ce cadrage nous positionnerons en conclusion les différentes contributions de ce numéro.

\section{L'agriculture, nouvel enjeu mondial}

Un certain nombre d'événements ont remis ces dernières années la question de l'agriculture au tout premier plan des débats publics. Non pas que celleci ait été absente des préoccupations qui orientent les politiques publiques et les initiatives privées mais plutôt que ces préoccupations aient pris ces derniers temps une autre coloration. L'augmentation des besoins alimentaires à l'échelle planétaire dans un contexte de raréfaction des ressources naturelles (eau, pétrole, etc.), l'importance grandissante des préoccupations environnementales (diminution de la biodiversité, changement climatique, pollutions), ainsi que l'émer- gence de tensions dans l'accès au foncier dues à l'affectation à des fins autres qu'alimentaires de terres agricoles (filières énergétiques, urbanisation, grands aménagements), posent avec une acuité de plus en plus grande la question des agricultures à promouvoir pour nourrir la planète de manière durable. D'autres événements de nature plus conjoncturelle, comme l'irruption de crises sur la qualité sanitaire des aliments (crise de la "vache folle ") ou la forte variation des prix des matières agricoles liée non seulement aux aléas climatiques mais aussi à des mouvements spéculatifs sur ces marchés pouvant conduire à des émeutes de la faim, ont fait ressortir le caractère incertain et non maîtrisé des systèmes mis en place. L'agriculture est donc aujourd'hui confrontée à des défis majeurs. Ces défis renvoient non seulement à des questions de sécurité alimentaire et sanitaire, mais aussi à des interrogations sur l'aménagement des territoires (maintien des populations en zone rurale, sanctuarisation de certains espaces...), sur l'agencement au sein de ces territoires de modes de production différents (par exemple, agriculture familiale et agriculture capitalistique, ou encore, agriculture " agroécologique " et agriculture fortement " artificialisée ") et sur la vocation multifonctionnelle des espaces naturels et agricoles (productif, patrimonial, récréatif, symbolique...).

L'agriculture est donc le lieu de visées variées qui, pour partie d'entre elles, peuvent s'avérer contradictoires ou, pour le moins, difficilement conciliables. Ainsi, les propositions débattues sur la scène internationale oscillent entre la poursuite du modèle productiviste, impulsée par le mouvement de la révolution verte, et le développement de modèles alternatifs qui reposent sur les principes de l'agroécologie (de Schutter, 2011). La tension entre la nécessité de produire suffisamment - que ce soit pour nourrir l'ensemble des habitants de la planète ou être économiquement compétitif - et celle de respecter l'environnement anime ces débats autour de l'émergence d'une agriculture durable, l'usage même de la dénomination "agriculture écologiquement intensive "dans certaines enceintes académiques rendant cette tension particulièrement saillante. Les problèmes relatifs à l'agriculture sont donc plus complexes qu'antérieurement et impliquent dans leur compréhension et leur traitement une plus grande diversité d'acteurs possédant leur visée propre sur l'espace et les pratiques agricoles. Cet éclatement des visées est non seulement dû à l'émergence de nouveaux problèmes ou de nouveaux intérêts mais aussi à la constitution de nouveaux acteurs à même de les faire valoir (Lémery, 2006). En effet, le développement de l'agriculture, dans les pays du Nord comme du Sud, a été après la seconde guerre mondiale largement porté par des États qui se sont lancés, dans le cadre de démarches planifiées, dans des programmes d'aménagement et d'équipement des territoires et d'encadrement des agriculteurs. Mais à partir des années 1990, on assiste avec la montée du libéralisme à un retrait de ces États et à l'émergence ou au renforcement d'acteurs ou de collectifs d'acteurs, notamment privés (firmes, organisations non gouvernementales [ONG], organisations de producteurs, etc.), qui prennent en charge le traitement ou la défense de questions particulières. Cette diversité des visées conduit à des situations fortement conflictuelles ou de domination de certains acteurs sur d'autres, mais aussi à la mise en œuvre de démarches de négociation, voire de coopération.

\section{Des périodes et des formes d'organisation du conseil différentes}

Pendant la période de fort interventionnisme de l'État, le conseil a permis le développement de la production en favorisant dans une logique très descendante le transfert de connaissances et de techniques aux agriculteurs pour ce qu'il était convenu d'appeler la " modernisation de l'agriculture ". Dans les pays occidentaux, les États ont investi pour permettre la construction d'organisations de conseil qui ont pris des formes variées en fonction des différents contextes nationaux (Rémy et al., 2006). Dans les pays du Sud, l'établissement de coopérations bilatérales entre les États et l'implication 
d'instances internationales comme la Banque mondiale et la FAO ont largement contribué à la mise en place de structures de conseil sur la base de modèles standardisés. Les programmes "Training and Visit ", soutenus par la Banque mondiale dans de nombreux pays et promouvant une approche normative et descendante du conseil pour améliorer la production agricole, en sont des exemples forts.

Toutefois, et à des rythmes différenciés suivant les pays, les démarches de conseil se sont progressivement diversifiées pour répondre à des questions plus larges que celles touchant à la seule production agricole. Elles ont donné une place plus importante aux méthodes participatives en visant le renforcement des capacités des agriculteurs et en s'appuyant de manière plus volontariste sur les processus d'apprentissage (Röling et Jong, 1998). Parallèlement, dans les années 1990, le désengagement des États du secteur agricole a débouché sur une "privatisation" partielle de l'activité de conseil, cette privatisation ayant toutefois pris des formes très différentes suivant les pays (Rivera et Alex, 2004). Des organismes économiques, des ONG, des organisations de producteurs ou des collectivités locales se sont alors plus largement inscrits dans ce champ d'activité, en développant en leur sein des fonctions de conseil ou en se spécialisant dans ce domaine. De nouveaux agencements sont progressivement apparus entre le conseil public et le conseil privé. Cette évolution a conduit à une cohabitation sur un même territoire entre différents dispositifs de conseil aux objectifs divers.

Dans ce nouveau cadre, des initiatives visent à mieux structurer l'offre de conseil aux agriculteurs. Au niveau européen, par exemple, ont été mis en place des Systèmes nationaux de conseil agricole qui cherchent à lier les aides financières au respect des normes environnementales fixées par l'Union européenne (UE). De même, en 2010, le Global Forum on Rural Advisory Services (GFRAS), principalement porté par des organisations internationales, a été créé dans le but de permettre des échanges d'expériences sur le conseil dans les pays du Sud et de favoriser un nouvel investissement public dans ce domaine. Les évolutions perceptibles sur le terrain sont cependant diverses et encore incertaines. Des démarches qui considèrent le conseil comme un bien public cherchent à le rénover en redynamisant et en repositionnant les dispositifs publics et privés d'appui aux agriculteurs. D'autres le voient comme un processus d'accompagnement de dynamiques complexes qui opère au sein d'un système localisé d'innovations associant différentes catégories d'acteurs. D'autres encore le considèrent comme un moyen pour faire évoluer les exploitations en fonction d'injonctions définies par les marchés ou par certains secteurs de la société.

\section{Le conseil, fruit de choix politiques}

\section{sur le développement agricole}

Les évolutions du conseil ne sont donc pas le fruit du hasard mais des constructions sociales complexes. La structuration de l'espace du conseil au niveau d'un pays ou d'une région avec ses cohortes d'agents et leurs formes d'intervention spécifiques, a une dimension profondément politique. Des choix sont faits, que l'on laisse, par exemple, la main aux promoteurs des techniques pointues d'artificialisation du vivant ou que l'on favorise l'implication d'acteurs de l'aval cherchant à faire appliquer aux producteurs leurs cahiers des charges sur la qualité des produits; ou encore que des projets soient financés pour favoriser l'agriculture de conservation ou que des aides soient mises en place pour permettre le maintien sur un territoire donné de formes d'agriculture peu rentables. De la même façon, certains choix peuvent amener à confier l'activité de conseil à des organismes privés développant des prestations marchandes ou à faire assumer cette activité de conseil à des organismes dans le cadre d'un service public. Ces choix sont évidemment plus ou moins contraints selon le contexte économique et institutionnel du pays ou de la région, selon les rapports de force dans lesquels se trouvent engagés les différents acteurs du conseil et selon leur positionnement dans ce champ d'activité. Les éléments sur lesquels portent ces choix - méthode d'intervention, financement ou mécanismes d'orientation du conseil - interagissent également entre eux pour donner une configuration chaque fois spécifique au conseil.

Ces choix renvoient à la façon dont les acteurs locaux et la société dans son ensemble définissent la place de l'agriculture dans les territoires et pensent son développement. À notre sens, parler de "développement", c'est parler d'autre chose que de "progrès " ou d' "innovation ", bien que ces trois notions s'appuient sur l'idée d'une trajectoire temporelle conduisant à une situation plus favorable que la situation initiale. La notion de progrès incorpore l'idée d'une visée clairement définie, donc partagée par une large partie de la société, et vers laquelle il s'agit de s'acheminer pas à pas (il y a plus de 20 ans, on parlait ainsi de "progrès technique "). Son usage caractérise une période de grandes planifications des interventions techniques et une grande assurance dans l'effet globalement positif de ces techniques. Durant cette période, les connaissances "utiles" aux agriculteurs sont connues par les acteurs du conseil. La notion d'innovation - actuellement largement dominante - abandonne quant à elle l'idée d'une visée précise déterminée en dehors des acteurs concernés et ne préjuge plus du sens donné à l'innovation. Elle s'inscrit beaucoup mieux dans un contexte de production plus complexe et incertain, où c'est la profusion de nouvelles formes matérielles, sociales, intellectuelles qui doit permettre une adaptation permanente à un contexte qu'elles contribuent en même temps à modifier. Le développement n'est pas l'adoption par certains acteurs d'une visée définie par d'autres, pas plus qu'il n'est une conduite adaptative permanente liée au surgissement d'événements nouveaux (Lémery, 2009). Il correspond, dans un sens proche de la "capabilité " de Sen (1999), à une augmentation de la capacité des agriculteurs, d'une part à définir une visée qui leur soit propre et, d'autre part, à obtenir et à mettre en ouvre les moyens d'atteindre cette visée. Il contribue à améliorer leur autonomie et la maîtrise 
de ce qu'ils font. Toutefois ainsi énoncée, cette notion n'est pas non plus exempte d'ambiguiité. En effet, si des collectifs d'agriculteurs peuvent se constituer et s'inscrire dans un tel processus, rien ne garantit qu'ils contribuent à la construction d'un bien considéré comme commun par le reste de la société. En forçant le trait, on peut s'inquiéter des effets à long terme de choix de systèmes de production effectués par des collectifs d'agriculteurs, choix qui conduisent progressivement à une surexploitation des ressources naturelles ou à un déséquilibre entre des aménités positives et négatives en matière économique et environnementale auxquelles d'autres groupes sociaux peuvent être sensibles. On retrouve ici la dimension politique précédemment évoquée et une conception " négociée " du développement où la participation des agriculteurs tient une place importante dans l'élaboration des priorités. La définition de ce que doit être l'agriculture engage des acteurs assez divers, aux exigences variées, qu'ils soient agriculteurs ou non (Compagnone et al., 2009). C'est par leur capacité à arbitrer des points de vue différents que des orientations dans la manière de produire qui intègrent le souci du bien commun peuvent émerger.

\section{Le conseil agricole}

\section{entre}

\section{accompagnement}

\section{et encadrement}

En fonction des contextes, le conseil peut avoir des objectifs différents, parfois complémentaires, parfois contradictoires. Il peut rendre accessible aux agriculteurs une série d'interprétations ou d'analyses de leur situation, ou leur fournir divers outils, techniques, connaissances et manières de faire leur permettant d'améliorer leur système de production ou d'atteindre des visées qui leur sont propres, ou encore formuler des injonctions sur la manière de produire afin de répondre à des exigences économiques, techniques ou environnementales imposées par d'autres acteurs. Pour chaque objectif, des formes de conseil diffé- rentes sont mobilisées. Elles peuvent être directives et viser à dire aux agriculteurs ce qu'il faut faire et comment, ou être plus compréhensives et chercher à renforcer les capacités d'action et l'autonomie des personnes. Dans un sens on pourra parler " d'encadrement " des agriculteurs et dans l'autre "d'accompagnement ". Si dans un cas on peut se contenter d'opérer un transfert de connaissances, dans l'autre il s'agit de susciter un réel processus d'apprentissage (Röling et Groot, 1998). D'un extrême à l'autre, la participation des agriculteurs à l'identification des problèmes, à la production du conseil ou à l'évaluation de ses résultats peut être très variable. Mais, d'une manière ou d'une autre, le conseil est toujours " orienté " dans le sens où il constitue une proposition d'interaction et d'action. Il est pour les agriculteurs à la fois une ressource et une contrainte. Il les aide à savoir ce qu'il faut faire et comment, mais, en même temps, il les " oblige ", rendant certaines façons de faire obsolètes, d'autres obligatoires, empêchant parfois le développement de formes alternatives d'agriculture. Les agriculteurs qui en bénéficient se trouvent donc plus ou moins tributaires de ce cadre selon leur capacité à mettre en perspective des ressources informatives et cognitives d'origines différentes, que ces ressources proviennent du milieu technique ou d'autres agriculteurs. En fonction des objectifs du conseil et de la manière de l'apporter tous les agriculteurs n'expriment pas le même intérêt et tous n'ont pas le même accès à ce service. Vont ainsi jouer leur appartenance ou adhésion à des organisations apportant du conseil et le coût qu'ils devront supporter pour pouvoir en bénéficier.

Dans les faits, le conseiller est de plus en plus obligé aujourd'hui de tenir ensemble des logiques d'action différentes (Compagnone et al., 2008). De nouvelles exigences techniques, économiques, sociales ou environnementales sont adressées aux agriculteurs. Elles se traduisent par des cadres réglementaires de plus en plus étoffés portés par la puissance publique ou prennent la forme de cahiers des charges de plus en plus élaborés développés par des acteurs privés (industrie, distribution, consommation). Dans ce sens, un processus de rationalisation et de normalisation de l'agriculture est en cours qu'il s'agit pour le conseiller de relayer, bien que ce processus puisse être aussi source d'exclusion de certains agriculteurs. Par ailleurs, de nouvelles questions se posent sur les modes de production à mettre en œuvre. Les réponses n'étant pas déjà disponibles, il est aussi nécessaire pour le conseiller d'accompagner les agriculteurs, dans le cadre d'un processus d'innovation, pour leur permettre de produire des connaissances à la fois adaptées aux situations locales et cohérentes avec les nouvelles exigences de production. L'enjeu est ici de leur permettre non seulement de changer de pratiques de manière maîtrisée mais aussi de pouvoir identifier, recenser et faire circuler dans un espace plus large les connaissances produites localement, en les hybridant, si nécessaire, avec des savoirs scientifiques. Cette logique réglementaire et cette logique d'innovation portées dans le conseil peuvent bien évidemment être antagonistes et obligent le conseiller à composer avec des registres d'action différents.

À ces deux logiques viennent s'en adjoindre deux autres qui portent quant à elles plus spécifiquement sur la capacité technique des conseillers. Un conseil standard auprès de l'ensemble des agriculteurs est de moins en moins tenable, une partie de ces derniers gagnant en compétences et formulant des demandes précises et pointues. Les conseillers sont alors amenés à intervenir pour apporter de manière ciblée et spécifique une réponse à ces questions. Leur intervention étant parfaitement calibrée en termes d'objet et de durée, elle peut être évaluée et s'inscrire dans le cadre d'un échange marchand, largement encouragé dans un contexte de libéralisation de l'économie. Toutefois cette capacité technique peut aussi être mise en œuvre par les conseillers non pas auprès des agriculteurs mais auprès de leurs employeurs ou des pouvoirs publics. Leur travail consiste alors à apporter aux personnes à même de prendre des décisions des éléments de connaissance sur leurs domaines techniques ou sur les pratiques et caractéristiques des agriculteurs qu'ils conseillent. Ils interviennent comme experts dans un processus de décision. Dans ces deux types de démarches, une logique de service et une logique d'expertise se révèlent. Enfin, les conseillers étant 
impliqués dans d'autres sphères sociales que celle des producteurs agricoles, ils sont amenés à jouer le rôle de médiateurs entre les agriculteurs et les acteurs de ces autres sphères. Ils rentrent dans des logiques de négociation. On voit donc apparaître à travers la description de ces cinq logiques des types d'activité des conseillers fortement différenciés.

\section{Les objets d'attention dans les politiques publiques \\ et la recherche}

Face à cette évolution du conseil, quels sont aujourd'hui les positions de la puissance publique et les objets sur lesquels la recherche centre ses travaux? Au niveau de la puissance publique, le débat se déplace progressivement, du renouvellement des dispositifs de conseil aux agriculteurs, portés par des organisations spécialisées, publiques ou privées, vers le renforcement des systèmes d'innovation, via des politiques adaptées. Un système d'innovation peut être défini comme un réseau d'organisations, d'entreprises et d'individus produisant de nouveaux produits, de nouveaux processus ou de nouvelles formes d'organisation qui sont ensuite utilisés dans des activités économiques et sociales, en lien avec des institutions et des politiques qui affectent leurs comportements et leurs performances (World Bank, 2006). Dans une littérature qui prend de plus en plus d'importance, le conseil agricole est alors considéré comme partie intégrante d'un système d'innovation (Birner et al., 2009) et les acteurs du conseil, c'est-à-dire les organisations fournissant du conseil et leurs agents, se voient attribuer un rôle de médiation entre les agriculteurs et les organismes économiques ou les collectivités territoriales. La logique de négociation est alors dominante. Dans une telle perspective, l'agriculteur n'est plus au centre des préoccupations des conseillers, ni la cible prioritaire des dispositifs d'encadrement ou d'accompagnement. De plus, d'autres acteurs que les conseillers sont amenés à jouer ce rôle de médiation (Klerkx et Leeuwis, 2008). Les décideurs politiques ou les bailleurs de fonds peuvent ainsi, pour favoriser l'innovation en agriculture, intervenir auprès de différents acteurs du système d'innovation sans forcément privilégier les acteurs du conseil (Klerkx et al., 2010). Renforcer une organisation de producteurs qui fournit des services à ses membres, dont du conseil, peut être ainsi considéré comme plus utile que d'appuyer une organisation de conseil qui travaille directement avec les producteurs.

En ce qui concerne la recherche, quelle place occupe-t-elle dans ce tableau ? Dans une logique réglementaire, elle joue toujours un rôle dominant dans la production de connaissances scientifiquement validées et dans l'élaboration de normes. Suivant un schéma très diffusionniste, elle fournit alors des références pour nourrir le conseil. En revanche, dans une logique d'innovation, elle contribue aussi à la production de connaissances en acceptant la confrontation des différents types de savoirs. Son apport dans l'innovation est alors plus difficile à caractériser mais essentiel pour promouvoir une agriculture durable (IAASTD, 2009). En ce qui concerne la thématique même du conseil, la recherche a développé un champ scientifique spécifique pour comprendre les dynamiques en cours et y contribuer. Les équipes de recherche sont localisées principalement en Europe, aux États-Unis et en Australie, au sein d'universités traitant de l'agriculture, et elles associent souvent dans leurs démarches activités de recherche sur le conseil et activités de conseil auprès d'agriculteurs. En France, la recherche sur cette thématique est plutôt concentrée au niveau de l'Institut national de la recherche agronomique (Inra) qui n'intervient pas directement dans le conseil. D'autres pays développent de manière de plus en plus importante des recherches sur le sujet, notamment l'Inde, le Pakistan ou le Nigeria. Enfin des organisations internationales comme l'International Food Policy Research Institute (IFPRI) et la Banque mondiale ont une production intellectuelle significative sur le sujet. Les questions traitées sont diverses et portent sur : i) le renouvellement des politiques d'intervention pour adapter le conseil aux nouveaux défis de l'agriculture ; ii) le fonctionnement des organisations du conseil dans le cadre d'une redéfinition du rôle du conseil public et du conseil privé ; iii) l'évolution des méthodes de conseil pour prendre en compte les nouvelles dimensions du développement et favoriser les processus d'apprentissage ; iv) l'adaptation des compétences des conseillers à des situations nouvelles, tant du point de vue des thématiques abordées que de la manière de produire le conseil ou de le faire financer ; et enfin v) la production de connaissances (entre chercheurs ou avec les producteurs) pour analyser et résoudre les problèmes auxquels sont confrontés les agriculteurs et les conseillers.

\section{Conclusion :}

\section{les apports}

\section{du numéro thématique}

\section{sur le conseil}

Les réflexions précédentes permettent de préciser les enjeux auxquels le conseil se trouve aujourd'hui confronté. L'ambition de ce numéro est de rendre compte des transformations qu'opère le conseil pour répondre à ces enjeux. Il vise à caractériser et à comprendre les déterminants des évolutions différenciées selon les situations mais aussi à analyser différentes démarches de conseil mises en œuvre dans ce nouveau contexte. Un accent particulier est mis sur l'évolution du métier des conseillers et sur les outils qu'ils mobilisent dans leur travail. Ce numéro tire son originalité de l'étendue des thématiques du conseil qu'il couvre et qui tiennent au questionnement présenté ci-dessus. Pour ce faire, il met en perspective aussi bien des situations de conseil de différents pays du Nord (France, autres pays d'Europe et Canada) que de pays du Sud (Chili, Argentine, Maroc, Bénin, Cameroun). De la même manière, il s'appuie sur des points de vue disciplinaires différents (économie, sciences de gestion, sociologie, agronomie, géographie).

Après un premier article dressant un panorama bibliographique des productions scientifiques réalisées dans le domaine du conseil depuis une dizaine d'années (Faure et al., 2011), trois autres contributions s'attacheront à rendre compte des politiques 
publiques mises en œuvre en matière de conseil et s'intéresseront aux conséquences de ces politiques dans l'accès au conseil des agriculteurs, que ce soit en Europe (Labarthe et al., 2011) ou en Amérique (Namdar et al., 2011 ; Gaboury-Bonhomme, 2011). Ces politiques se matérialisent dans la mise en place de dispositifs d'appui aux agriculteurs qui se déploient dans un espace social où d'autres, plus ou moins concurrents, sont déjà présents. Les articles suivants feront apparaître la nature de ces dispositifs et les stratégies des organisations qui les déploient, que celles-ci aient pour visée le développement (Faure et al., 2011 ; Djamen et al., 2011 ; Moumouni et al., 2011) ou la vente de services et de produits (Goulet, 2011). Les contributions se centrent ensuite sur les activités des conseillers, sur la manière dont ils les mènent, sur les ressources sur lesquelles ils s'appuient et sur les interactions qu'ils développent entre eux et avec les agriculteurs. Parmi ces contributions, certaines s'intéressent aux formes du métier de conseiller et à la manière dont il est exercé en Argentine (Albaladejo, 2011) et en France (Auricoste et al., 2011 ; Compagnone et al., 2011 ; Petit et $a l ., 2011$ ). D'autres se penchent sur les outils qui peuvent être produits pour apporter un conseil pertinent aux agriculteurs, que ces outils soient matériels (Le Gal et al., 2011) ou immatériels (Magne et al., 2011 ; Layadi et al., 2011).

\section{Références}

Albaladejo C, 2011. Transformations du consei agricole en région pampéenne argentine et recomposition de la profession "d'ingénieur agronome ". Cahiers Agricultures 20 : 387-94. doi : 10.1684/ agr.2011.0504.

Auricoste C, Albaladejo C, Barthe L, Couix N, Duvernoy I, Girard N, et al., 2011. Accompagner I'activité agricole dans les territoires : au carrefour entre le développement sectoriel et le développement territorial. Cahiers Agricultures 20 : 395-9. doi : 10.1684/agr.2011.0502.

Birner R, Davis KE, Pender J, Nkonya E, Anandajayasekeram P, Ekboir J, et al., 2009. From best practice to best fit: a framework for designing and analyzing pluralistic agricultural advisory services worldwide. Journal of Agricultural Education and Extension $15: 341-55$.

Compagnone C. Petit S. Lémery B, 2008. A new role of mediator for extension services: a challenge for the Chambers of agriculture in France. Journal of Extension Systems (2) : 16-29.

Compagnone C, Auricoste C, Lémery B, eds, 2009. Conseil et développement en agriculture : quelles nouvelles pratiques? Dijon; Versailles : Educagri éditions ; éditions Quae.

Compagnone C, Golé S, 2011. La reconfiguration de I'espace social du conseil en agriculture : le cas du conseil phytosanitaire en viticulture bourguignonne. Cahiers Agricultures 20: 400-5. doi : 10.1684/agr.2011.0505.

De Schutter O, 2011. Agroécologie et droit à I'alimentation. Rapport présenté à la $16^{\mathrm{e}}$ session du Conseil des droits de l'homme de l'ONU, A/HRC/ 16/49, Genève, 8 mars 2011. http://www.srfood. org/images/stories/pdf/officialreports/20110308 a-hrc-16-49 agroecology fr.pdf.

Djamen Nana P, Havard M, Wey J, 2011. L'évolution du conseil agricole au Nord Cameroun, source d'incertitudes pour les conseillers. Cahiers Agricultures 20 : 370-5. doi : 10.1684/agr.2011.0506.

Faure G, Desjeux Y, Gasselin P, 2011. Revue bibliographique sur les recherches menées dans le monde sur le conseil en agriculture. Cahiers Agricultures 20 : 327-42. doi : 10.1684/agr. 2011.0510.

Faure G, Rebuffel P, Violas D, 2011. Une analyse systémique des dispositifs de conseil à l'exploitation familiale en Afrique de l'Ouest. Cahiers Agricultures 20: 364-9. doi : 10.1684/agr. 2011.0507.

Gaboury-Bonhomme ME, 2011. Évolution de la gouvernance et des politiques de services-conseils agricoles au Québec (Canada). Cahiers Agricultures 20 : 359-63. doi : 10.1684/agr.2011.0513.

Goulet F, 2011. Accompagner et vendre. Les firmes de l'agrofourniture dans I'innovation et le conseil en agriculture. Cahiers Agricultures 20 : 382-6. doi : 10.1684/agr.2011.0503.

IAASTD, 2009. Agriculture at a crossroads. Synthesis report. Washington (DC) : Island Press.

Klerkx L, Leeuwis C, 2008. Matching demand and supply in the agricultural knowledge infrastructure: experiences with innovation intermediaries. Food Policy $33: 260-76$

Klerkx L, Aarts, N, Leeuwis C, 2010. Adaptive management in agricultural innovation systems: the interactions between innovation networks and their environment. Agricultural Systems 103 : 390400.

Labarthe P, Laurent C, 2011. Économie des services et politiques publiques de conseil agricole. Cahiers Agricultures 20 : 343-51. doi : 10.1684/ agr.2011.0508.
Layadi A, Faysse N, Dumora C, 2011. Les organisations professionnelles agricoles locales, partenaires pour renforcer le dialogue technique ? Une expérience avec des éleveurs de bovins au Maroc. Cahiers Agricultures 20 : 428-33. doi : 10.1684/agr.2011.0511.

Le Gal PY, Andrieu N, Dugué P, Kuper M, Taher Sraïri M, 2011. Des outils de simulation pour accompagner des agroéleveurs dans leurs réflexions stratégiques. Cahiers Agricultures 20:413 20. doi : 10.1684/agr.2011.0509.

Lémery B, 2006. Nouvelle agriculture, nouvelles formes d'exercice et nouveaux enjeux du conseil aux agriculteurs. In : Rémy J, Brives H, Lémery $B$, eds. Conseiller en agriculture. Dijon; Versailles : Educagri éditions ; éditions Quae.

Lémery B, 2009. Le développement agricole à l'épreuve d'un nouveau régime de production des savoirs sur le vivant. In : Hervieu B, Hubert B, eds. Sciences en campagne. Regards croisés, passés et à venir. La Tour d'Aigues: éditions de I'Aube.

Magne MA, Cerf M, Ingrand S, 2011. Comment les éleveurs choisissent-ils et utilisent-ils des informations pour conduire leur exploitation? Quelques enseignements pour les conseillers. Cahiers Agricultures 20: 421-7. doi : 10.1684/agr. 2011.0512

Moumouni I, Nouatin GS, Baco MN, 2011. Du système formation et visites au conseil à l'exploitation agricole familiale au Bénin: rupture ou continuité ? Cahiers Agricultures 20: 376-81. doi : 10.1684/agr.2011.0514.

Namdar-Irani M, Sotomayor O, 2011. Le conseil agricole au Chili face à la diversité des agriculteurs. Cahiers Agricultures 20 : 352-8. doi : 10.1684/ agr.2011.0515.

Petit S, Compagnone C, Lémery B, Kockmann F, Moretty P, 2011. Les chambres d'agriculture francaises face à la marchandisation du conseil aux agriculteurs. Cahiers Agricultures 20 : 406-12. doi : 10.1684/agr.2011.0516.

Rémy J, Brives H, Lémery B, eds. Conseiller en agriculture. Dijon ; Versailles : Educagri éditions ; éditions Quae.

Röling N, Jong FD, 1998. Learning: shifting paradigms in education and extension studies. Journal of Agricultural Education and Extension $5: 143-61$.

Röling N, Groot A, 1998. Contemplating alternatives - A comparative framework for thinking about extension. Agriculture + Rural Development 5 : 11-3.

Rivera WM, Alex G, 2004. Extension system reform and the challenges ahead. Journal of Agricultural Education and Extension 10 : 23-36.

Sen A, 1999. Development as freedom. Oxford: Oxford University Press.

World Bank, 2006. Enhancing agricultural innovation: How to go beyond the strengthening of research systems. Washington (DC) : World Bank. 